\title{
A catalogue of isolated massive white dwarfs ${ }^{\star}$
}

\section{Mass distribution of massive stars}

\author{
M. Należyty ${ }^{1}$ and J. Madej ${ }^{2}$ \\ 1 Astronomical Observatory, University of Warsaw, Al. Ujazdowskie 4, 00-478 Warsaw, Poland \\ 2 N. Copernicus Astronomical Center, Bartycka 18, 00-716 Warsaw, Poland
}

Received 9 April 2003 / Accepted 15 January 2004

\begin{abstract}
We present in this paper a catalogue of 112 massive isolated white dwarfs, with masses $M>0.8 M_{\odot}$. Mass determinations and other parameters of white dwarfs were compiled from the available literature. For each star we present averaged values of mass, effective temperature, logarithm of surface gravity $\log g$, radius, distance, and the surface magnetic field for magnetic white dwarfs. The mass distribution of our sample is a slowly decreasing continuum function for masses larger than $0.9 M_{\odot}$, with an overlapping secondary maximum at $1.04 M_{\odot}$. We conclude that the mass distribution of known massive magnetic white dwarfs is flat, whereas nonmagnetic WDs exhibit a steeper mass distribution towards the highest masses. The secondary maximum at $1.04 M_{\odot}$ is caused exclusively by nonmagnetic white dwarfs. We note that the 4 most massive stars with masses $M \geq 1.3 M_{\odot}$ are magnetic white dwarfs. Our results show also, that the occurrence of magnetism in massive white dwarfs does not depend on the cooling age (above $T_{\text {eff }}=5000 \mathrm{~K}$ ).
\end{abstract}

Key words. catalogs - stars: white dwarfs

\section{Introduction}

The masses of white dwarf stars are always smaller than the Chandrasekhar mass, which is equal to 1.44 solar mass in the case of hydrogen non-rotating objects. It is well known, however, that the mass distribution of isolated white dwarfs exhibits peak at mass $\approx 0.60 M_{\odot}$ (Weidemann 1990), with a substantial number of known objects with higher masses. The analysis of recently obtained homogeneous samples of isolated white dwarfs has led to a slightly different peak mass value. Bergeron et al. (1992, hereafter BSL) have analyzed the sample of 129 DA white dwarfs, and determined their masses by means of fitting hydrogen Balmer line profiles. They obtained a peak mass value of $0.562 M_{\odot}$. Liebert \& Bergeron (1995) analyzed 200 white dwarfs from the Palomar Green survey (Green et al. 1986), with peak mass $0.56 M_{\odot}$. However, the above surveys did not reach to much higher masses, and were only sparsely populated by white dwarfs $>1 M_{\odot}$. Only recently Marsh et al. (1997a,b) determined the masses (and also other stellar parameters) of an extensive set of white dwarfs selected from the ROSAT all-sky survey in the extreme ultraviolet (EUV). They distinguished between the populations of normal $\left(\approx 0.6 M_{\odot}\right)$ and massive $\left(>1.0 M_{\odot}\right)$ white dwarf stars.

Send offprint requests to: M. Należyty,

e-mail: nalezyty@astrouw.edu.pl

* The full Table 1 is only available in electronic at the CDS via anonymous ftp to cdsarc.u-strasbg. fr $(130.79 .128 .5)$ or via http://cdsweb.u-strasbg.fr/cgi-bin/qcat?J/A+A/420/507
Extensive determinations of the WD mass distribution were also presented in recent papers by Vennes et al. (1997a,b, 1998), and Vennes (1999), which were based on the Extreme Ultraviolet Explorer (EUVE) observations.

In this paper we present and discuss the catalogue and the mass distribution of massive white dwarfs with masses $M>0.8 M_{\odot}$. Our research is based on mass determinations available in the existing literature. Investigation of the mass distribution of white dwarfs on the massive branch can put significant constraints on both early and late stages of stellar evolution, including star forming stages in the Galactic disc. The limiting mass $0.8 M_{\odot}$ in this paper has been chosen arbitrarily. Masses presented in the following sections represent a highly inhomogeneous sample, since we intended to collect as many massive white dwarf stars as possible.

Masses of isolated white dwarfs are probably correlated with the chemical composition of their cores. White dwarf cores are generally believed to be built of carbon and oxygen. However, there exists a number of papers, which study the mass-radius relations, cooling sequences, and photometric colors for helium-core white dwarfs (Serenelli et al. 2001, 2002; Rohrmann et al. 2002), which are located at the low mass end of mass distribution. Similarly white dwarfs with mass higher than the average mass can be correlated with the population of magnesium-core or iron-core stars studied by Provencal et al. (1998), Provencal \& Shipman (1999), Panei et al. (2000), Madej et al. (2003). The present catalogue listing the 
massive isolated white dwarfs probably contains white dwarfs with cores consisting of heavier elements.

Masses of isolated white dwarfs are usually determined with the help of spectral analysis. The observed visual spectra can be fitted with theoretical spectra to determine effective temperatures $T_{\text {eff }}$ and surface gravities $\log g$ for some assumed chemical composition, mostly pure hydrogen. A classical paper by Shipman (1979) explained the method of radius $R$ and mass $M$ determination from known values of $T_{\text {eff }}, \log g$, distance $d$, and visual magnitude $m_{\mathrm{V}}$, based on some reasonable grid of synthetic spectra. Nowadays there exists three principal methods of mass and radius determination of isolated white dwarfs, which are used depending on the exact set of available observational parameters, which include also estimates of the gravitational redshift (cf. discussion in Schmidt 1997).

Other techniques of mass determination result from orbital solutions in isolated binaries containing a white dwarf (Sirius B, Procyon B, for instance). A review of the various methods of mass determination has been given by BSL (1992), cf. also Koester (2002).

In the present paper, we disregard the differences between particular methods of $M$ and $R$ determination, and collect isolated massive white dwarfs in a single set of stars. In this way we attempt to investigate the most numerous set of star which is actually available, and avoid random fluctuations of small numbers of binned stars. It is necessary to stress here that white dwarfs in interacting binaries were not included in our catalog.

\section{The catalogue}

In this paper we present an analysis of the catalogue of all massive white dwarfs, compiled from the available literature. Our catalogue consists of 112 white dwarf stars, both magnetic and nonmagnetic WDs. The full catalogue is not printed in this paper, and available in electronic form at the CDS. A shortened version of the catalogue is presented in Table 1.

The data for each star were compressed into a single row. The columns of Table 1 list the following data: WD designation by its equatorial coordinates, name of the star, and values and errors of $T_{\text {eff }}, \log g, M / M_{\odot}$. The ninth and following columns give; radius $R$ in kilometers, mean surface magnetic field $B_{\mathrm{s}}$, polar field $B_{\mathrm{p}}$, distance $d$ in kiloparsecs, remarks, and a reference list.

In most cases the stellar parameters were independently determined by several authors. Values of $T_{\text {eff }}, \log g, M / M_{\odot}$, and remaining parameters presented in Table 1 are arithmetic averages of the individual data. The errors are just formal errors of the above averages. In this way we could neglect error determinations given in individual papers. Parameters of white dwarfs determined in a single paper have not been assigned any error estimates in Table 1.

\section{The mass distribution of white dwarf stars}

The mass distribution of isolated white dwarfs exhibits a relatively narrow peak at about $0.6 M_{\odot}$. Precise values of the peak mass were determined to be $0.603 M_{\odot}$ (Weidemann \& Koester 1984), $0.571 M_{\odot}$ (McMahan 1989),

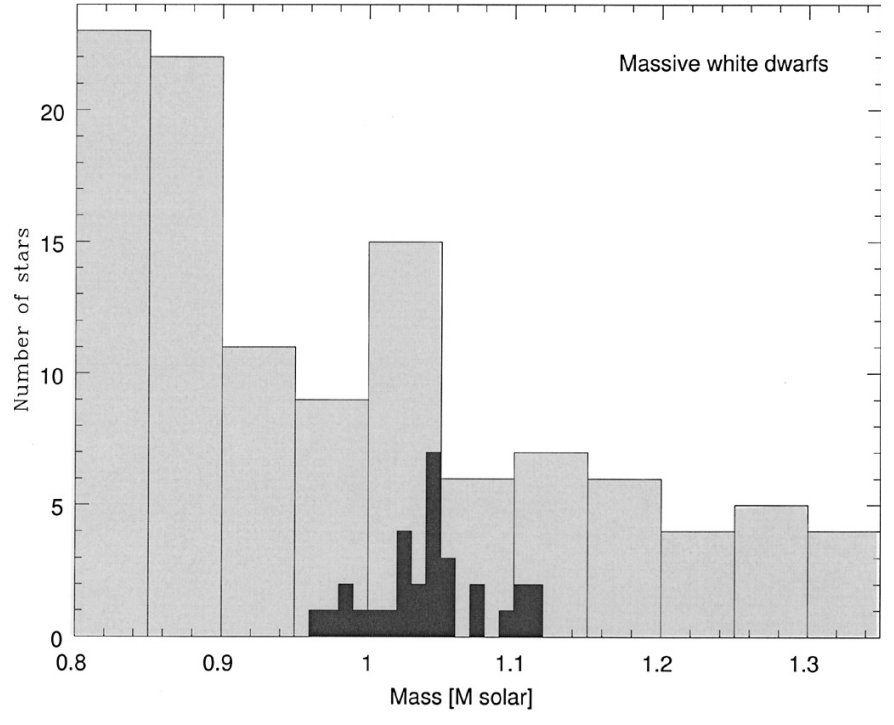

Fig. 1. Mass distribution for all 112 massive white dwarf stars of our catalogue (gray scale). The wide-binned histogram shows the local maximum of the mass distribution in the range 1.0-1.05 $M_{\odot}$. The inserted dark tone histogram with finer resolution of $0.01 M_{\odot}$ clearly suggests that the local maximum of the WD mass distribution is located at $\approx 1.04 M_{\odot}$.

$0.562 M_{\odot}$ (BSL 1992), $0.56 M_{\odot}$ (Liebert \& Bergeron 1995), $0.570 M_{\odot}$ (Finley et al. 1997), and $\approx 0.55 M_{\odot}$ (Marsh et al. 1997a,b).

The shape of the mass distribution also exhibits a distinct tail towards higher masses, which hardly any of the above papers reproduced satisfactorily. Weidemann \& Koester (1984) did not include white dwarfs of masses higher than 1.0 $M_{\odot}$, and there are only few such massive stars in other early papers presented in their list. Marsh et al. (1997) recognized the existence of a high-mass population $\left(>0.8 M_{\odot}\right)$, consisting a total of 13 white dwarfs only. Small sample of massive white dwarfs in that paper did not make it possible to investigate any details of the mass distribution in that region. In spite of this, Marsh et al. (1997) suggested that the massive white dwarf stars form a second population, clearly differing from the main population with a mass peak at about $0.6 M_{\odot}$, which probably were formed by coalescence of normal white dwarfs in close binary system.

\section{The tail of the massive WD distribution}

White dwarfs and their averaged masses collected in Table 1 form a rather inhomogeneous sample, as we did not discriminate between various methods of mass determination. This was done to increase the total number of massive white dwarfs in the sample. In this way we attempt to minimize the uncertainties and fluctuations caused by the very small number of massive stars available in previous investigations.

Figure 1 presents the mass distribution of all 112 isolated white dwarfs, with averaged masses compiled in Table 1. The main histogram consists of bins with $0.05 M_{\odot}$ width which shows, that above $0.9 M_{\odot}$ the number of stars per bin continuously decreases towards higher masses, and only the single bin 1.0-1.05 $M_{\odot}$ contains a significantly larger number of stars 
Table 1. Catalogue of massive white dwarfs.

\begin{tabular}{|c|c|c|c|c|c|c|c|c|c|c|c|c|c|}
\hline WD & Name & $T_{\text {eff }}$ & $\Delta T_{\text {eff }}$ & $\log g$ & $\Delta \log g$ & $M / M_{\odot}$ & $\Delta M / M_{\odot}$ & $R$ & $B_{\mathrm{s}}$ & $B_{\mathrm{p}}$ & $\bar{d}$ & Rem. & References \\
\hline $0000-345$ & GR406 & 7000 & & & & 0.92 & & & 70 & & & $\mathrm{~m}$ & 5 \\
\hline $0003+436 \mathrm{~J}$ & RE0003+433 & 45107 & 1362 & 9.01 & 0.15 & 1.21 & 0.06 & 3907 & & & 101 & & $1 \mathrm{a}, 1 \mathrm{~b}, 2,3,4$ \\
\hline $0008+330$ & HSO008+3302 & 10300 & & 8.35 & & 0.83 & & 6960 & & & 85 & & 14 \\
\hline $0009+501$ & GR381 & 6400 & & & & 0.89 & & & & 70 & & $\mathrm{~m}$ & 17 \\
\hline $0022+274$ & LP349-013 & 25000 & & & & 0.862 & & & & & 29 & $\mathrm{~b}$ & 8 \\
\hline $0033+016$ & EG004 & 10700 & & 8.66 & & 1.02 & & 5440 & & & 32.9 & & 6 \\
\hline $0041+092$ & $\mathrm{BD}+08^{\circ} 102$ & 28960 & 50 & 8.50 & & 0.90 & & 6180 & & & 55 & $\mathrm{~b}$ & 27,28 \\
\hline $0046+051$ & EG005 & 6770 & & 8.40 & & 0.83 & & 6620 & & & 4.3 & & 6 \\
\hline $0115+159$ & EG009 & 9800 & & 8.38 & & 0.82 & & 6740 & & & 15.4 & & 6 \\
\hline $0136+251$ & PG0136+251 & 39465 & 294 & 9.01 & 0.04 & 1.21 & 0.03 & 3830 & & & 80 & $\mathrm{~m}$ & $1 \mathrm{a}, 1 \mathrm{~b}, 2,3,4,18$ \\
\hline $0146+072$ & HS0146+0723 & 25000 & & 8.27 & & 0.80 & & 7520 & & & 210 & & 14 \\
\hline $0235-125$ & PHL 1400 & 32018 & 252 & 8.49 & 0.05 & 0.95 & 0.03 & 6170 & & & 66 & & $1 \mathrm{a}, 1 \mathrm{~b}, 2,3,12$ \\
\hline $0239+500 \mathrm{~J}$ & EUVE J0239+500 & 34211 & 389 & 8.517 & 0.043 & 0.96 & 0.02 & 6130 & & & 96 & & 2,3 \\
\hline $0317-853$ & EUVE J0317-855 & 43210 & 3290 & 9.19 & 0.30 & 1.34 & 0.01 & 2408 & 505 & 395 & & $\mathrm{mbc}$ & $4,5,17,22,23,24,25$ \\
\hline 0346-011 & GD 50 & 41743 & 736 & 9.12 & 0.04 & 1.25 & 0.03 & 3520 & & & 29 & & $1 \mathrm{a}, 1 \mathrm{~b}, 2,4,7,12,13$ \\
\hline $0347+171$ & V471 Tau & 34060 & 580 & 8.40 & 0.14 & 0.90 & 0.07 & 6240 & & & 47 & $\mathrm{bc}$ & $1 \mathrm{a}, 1 \mathrm{~b}, 29,30,31$ \\
\hline $0349+247$ & EG025 & 32180 & 320 & 8.69 & 0.05 & 1.046 & 0.012 & 5081 & & & & & $7,9,11,19,32$ \\
\hline $0352+049$ & KUV03520+0500 & 36900 & 500 & 8.71 & 0.15 & 1.05 & 0.08 & 5280 & & & 106 & & 4,14 \\
\hline $0406+169$ & EG029 & 15190 & & 8.30 & & 0.806 & 0.013 & 7300 & & & 53.2 & & $8,9,11,16$ \\
\hline HD27483 & & 22000 & & 8.5 & & 0.95 & & 6300 & & & 46 & bt & 27 \\
\hline 0443-037J & EUVE J0443-037 & 68740 & 3600 & 8.946 & 0.174 & 1.25 & 0.04 & 3970 & & & 144 & & $2,3,4$ \\
\hline $0518-105$ & RE0521-102 & 32727 & 323 & 8.67 & 0.02 & 1.04 & 0.01 & 5380 & & & 99 & $\mathrm{~b}$ & $1 \mathrm{a}, 1 \mathrm{~b}, 2,3,12$ \\
\hline $0531-022$ & EUVE J0534-022 & 29867 & 133 & 8.587 & 0.054 & 1.00 & 0.02 & 5760 & & & 101 & & 2,3 \\
\hline $0548-001$ & EG248 & 6400 & 100 & 8.32 & & 0.81 & 0.03 & 7040 & 8 & & 11.1 & $\mathrm{~m}$ & 5,6 \\
\hline 0557-165J & 1RXSJ0557.0-1635 & 56820 & & 8.88 & & 1.15 & & 4490 & & & 309 & & 4 \\
\hline 0630-050 & RE0632-050 & 43029 & 686 & 8.32 & 0.13 & 0.81 & 0.07 & 7790 & & & & & $1 \mathrm{a}, 1 \mathrm{~b}, 2,3$ \\
\hline $0633+200 \mathrm{~J}$ & $0630+200$ & 75792 & & 8.398 & & 0.947 & & 7090 & & & & & 3 \\
\hline $0642-166$ & Sirius B & 24700 & 100 & 8.61 & 0.04 & 1.02 & 0.01 & 5670 & & & 2.64 & $\mathrm{~b}$ & $1 \mathrm{a}, 1 \mathrm{~b}, 15,26$ \\
\hline $0644+025$ & GR484 & 7410 & & 8.66 & & 1.01 & & 5420 & & & 18.5 & & 6 \\
\hline $0653-564$ & EUVE J0653-564 & 35200 & & 8.88 & & 1.15 & 0.01 & 4490 & & & 107 & & 2,4 \\
\hline $0654+027$ & EG181 & 9450 & & 8.51 & & 0.91 & & 6110 & & & 38.5 & & 6 \\
\hline 0659-063 & LHS1892 & 6520 & & 8.71 & & 1.04 & & 5190 & & & 12.3 & & 6 \\
\hline 0701-587 & BPM18398 & 15701 & & 8.562 & & 0.944 & & 5861 & & & & & 13 \\
\hline 0729-384 & y Pup & 43200 & 200 & 8.5 & & 0.87 & 0.04 & 6100 & & & 172 & bt & 27,33 \\
\hline $0730+487$ & GD 86 & 15510 & & 8.49 & & 0.90 & & 6220 & & & & & 7 \\
\hline 0743-391J & EUVE J0743-391 & 40200 & & 8.66 & & 1.04 & 0.01 & 5500 & & & 147 & & 2 \\
\hline $0816+376$ & GD 90 & 11000 & & & & 0.86 & & & 8 & & & $\mathrm{~m}$ & 5 \\
\hline $0823-253$ & 1RXSJ0823.6-2525 & 43200 & & 9.02 & & 1.21 & 0.01 & 3910 & 3 & & 105 & $\mathrm{~m}$ & 4,34 \\
\hline $0827+328$ & EG249 & 7270 & & 8.39 & & 0.85 & & 6780 & & & 22.3 & & 6 \\
\hline $0836+197$ & LB 5893 & 21620 & 310 & 8.45 & & 0.916 & 0.007 & 6550 & & & 174 & & $8,9,16$ \\
\hline $0836+199$ & EG060 & 14060 & & 8.34 & & 0.864 & 0.021 & 7240 & & & & & 9 \\
\hline $0853+163$ & GR904 & 2000 & & & & 0.83 & & & 3 & & & $\mathrm{~m}$ & 5 \\
\hline $0856+331$ & EG182 & 10390 & & 8.84 & & 1.11 & & 4610 & & & 20.5 & $\mathrm{~b}$ & 6 \\
\hline $0912+536$ & EG250 & 7580 & 420 & 8.28 & & 0.87 & 0.12 & 7230 & 70 & & 10.3 & $\mathrm{~m}$ & 5,6 \\
\hline $0913+442$ & EG064 & 8620 & 130 & 8.24 & 0.05 & 0.826 & 0.093 & 7760 & & & 28.9 & $\mathrm{bp}$ & 6,11 \\
\hline 0916-197J & EUVE J0916-197 & 56400 & & 9.12 & 0.2 & 1.29 & 0.02 & 3600 & & & 164 & $\mathrm{~b}$ & 2,4 \\
\hline $0930+294$ & GR324 & 8330 & & 8.38 & & 0.84 & & 6820 & & & 32.1 & & 6 \\
\hline $0943+472$ & HS0943+4724 & 16000 & & 8.75 & & 1.07 & & 5000 & & & 120 & & 14 \\
\hline $0945+245.1$ & LB11146A & 14500 & & 8.5 & & 0.91 & & 6200 & & & 40 & $\mathrm{~b}$ & 35 \\
\hline $0945+245.2$ & LB11146B & 16000 & & 8.5 & & 0.99 & 0.09 & 6100 & 375 & 670 & 40 & $\mathrm{mb}$ & $5,17,35$ \\
\hline $0946+485$ & HS0946+4848 & 11700 & & 8.69 & & 1.04 & & 5300 & & & 80 & & 14 \\
\hline $0946+534$ & EG251 & 8760 & & 8.45 & & 0.87 & & 6400 & & & 23.0 & & 6 \\
\hline $0949+494$ & HS0949+4935 & 15000 & & 8.39 & & 0.86 & & 6800 & & & 190 & & 14 \\
\hline $0957+854 \mathrm{~J}$ & EUVE J0957+854 & 51636 & 325 & 8.32 & 0.06 & 0.83 & 0.02 & 7470 & & & 139 & & 2,12 \\
\hline $1015+014$ & PG1015+015 & 14000 & & & & 1.03 & & & 85 & & & $\mathrm{~m}$ & 5 \\
\hline $1017+366$ & GD 116 & 16000 & & & & 0.89 & & & 56 & & & $\mathrm{~m}$ & 5,37 \\
\hline $1024-303 \mathrm{~J}$ & RE1024-302 & 35710 & 520 & 8.95 & 0.15 & 1.13 & 0.06 & 4520 & & & 64 & $\mathrm{~b}$ & $1 \mathrm{a}, 1 \mathrm{~b}, 3,4$ \\
\hline $1031+234$ & TON 527 & 25000 & & & & 1.11 & & & 500 & & & $\mathrm{~m}$ & 5 \\
\hline $1036-204$ & GR535 & 7500 & & & & 1.34 & & & 150 & & & $\mathrm{~m}$ & 5 \\
\hline $1038+633$ & PG1038+634 & 24800 & & 8.39 & & 0.85 & & 6780 & & & & & 7 \\
\hline $1052+273$ & GD 125 & 23064 & 314 & 8.340 & 0.071 & 0.814 & 0.047 & 7031 & & & & & 7,13 \\
\hline $1055-072$ & EG074 & 7420 & & 8.42 & & 0.85 & & 6550 & & & 12.2 & & 6 \\
\hline
\end{tabular}


Table 1. continued.

\begin{tabular}{|c|c|c|c|c|c|c|c|c|c|c|c|c|c|}
\hline WD & Name & $T_{\text {eff }}$ & $\Delta T_{\text {eff }}$ & $\log g$ & $\Delta \log g$ & $M / M_{\odot}$ & $\Delta M / M_{\odot}$ & $R$ & $B_{\mathrm{s}}$ & $B_{\mathrm{p}}$ & $d$ & Rem. & References \\
\hline $1102+748$ & GD 466 & 19800 & & 8.37 & & 0.83 & & 6850 & & & & & 7 \\
\hline $1127-311.1$ & ESO439-162 & 5400 & & & & 1.13 & & & 67 & & & $\mathrm{mb}$ & 5 \\
\hline $1134+300$ & GD 140 & 21470 & 220 & 8.46 & 0.02 & 0.87 & 0.03 & 6310 & & & 15.3 & & $3,7,15$ \\
\hline $1136-285$ & ESO439-026 & 4490 & & 9.02 & & 1.19 & & 3880 & & & 40.8 & & 6 \\
\hline $1215+323$ & EG089 & 7100 & & 8.68 & & 1.02 & & 5320 & & & 31.1 & & 6 \\
\hline $1236-495$ & LTT 4816 & 12210 & 340 & 8.70 & 0.04 & 1.03 & 0.02 & 5250 & & & 16.4 & & $6,10,13$ \\
\hline $1241+482$ & HS1241+4821 & 14800 & & 8.54 & & 0.95 & & 6100 & & & 90 & & 14 \\
\hline $1309+853$ & GR436 & 5600 & & & & 0.83 & & & 15 & & & $\mathrm{~m}$ & 5 \\
\hline $1334-160$ & EG101 & 18790 & 210 & 8.32 & & 0.811 & 0.010 & 7180 & & & & bp & 7,11 \\
\hline $1350-090$ & LP 907-037 & 9500 & & & & 0.98 & & & 0.1 & & & $\mathrm{~m}$ & 5 \\
\hline $1440+750 \mathrm{~J}$ & HS1440+7518 & 38260 & 1680 & 8.71 & 0.10 & 1.04 & 0.03 & 5470 & 7.7 & & 98 & $\mathrm{~m}$ & $2,4,12,17$ \\
\hline 1444-174 & LHS 378 & 4960 & & 8.37 & & 0.81 & & 6770 & & & 14.5 & & 6 \\
\hline $1446+286$ & TON 214 & 22839 & 102 & 8.327 & 0.034 & 0.815 & 0.006 & 7143 & & & & & 3,7 \\
\hline $1501+664$ & H $1504+65$ & 170000 & & 8.0 & & 0.86 & & 10700 & & & 630 & & 38 \\
\hline $1531-022$ & GD 185 & 18870 & & 8.39 & & 0.84 & & 6740 & & & & & 7 \\
\hline $1535-774 \mathrm{~J}$ & EUVE J1535-774 & 54800 & 3200 & 9.12 & 0.02 & 1.29 & 0.03 & 3580 & & & 107 & & 2,4 \\
\hline $1543-366$ & RE1546-364 & 45208 & & 8.875 & & 1.168 & & 4546 & & & 107 & & 3 \\
\hline $1609+135$ & EG117 & 9080 & & 8.75 & & 1.07 & & 5030 & & & 18.3 & & 6 \\
\hline $1609+631$ & PG1609+631 & 31033 & & 8.408 & & 0.893 & & 6806 & & & & & 3 \\
\hline $1625+093$ & GR327 & 6870 & & 8.44 & & 0.88 & & 6510 & & & 23.4 & & 6 \\
\hline $1642+413$ & RE J1643+411 & 27677 & 1139 & 8.376 & 0.156 & 0.858 & 0.105 & 6944 & & & & & 3,12 \\
\hline $1658+440$ & EUVE J1659+440 & 30410 & 100 & 9.36 & & 1.32 & 0.02 & 2780 & 2.3 & & 27 & $\mathrm{~m}$ & $2,4,5,12,17,18$ \\
\hline $1705+030$ & GR494 & 7050 & & 8.35 & & 0.80 & & 6870 & & & 17.5 & & 6 \\
\hline $1711+667 \mathrm{~J}$ & RE1711+664 & 47556 & 1434 & 8.957 & 0.067 & 1.191 & 0.050 & 4185 & & & & $\mathrm{~b}$ & 3,12 \\
\hline $1725+586$ & RE J1726+583 & 55100 & 1083 & 8.32 & 0.08 & 0.869 & 0.052 & 7410 & & & & & $1 \mathrm{a}, 1 \mathrm{~b}, 3,12$ \\
\hline $1727-360$ & EUVE J1727-360 & 32600 & & 9.04 & & 1.21 & & 3830 & & & & & 4 \\
\hline $1740-706$ & RE1746-703 & 47690 & 1120 & 8.95 & 0.04 & 1.16 & 0.03 & 4270 & & & & & $1 \mathrm{a}, 1 \mathrm{~b}, 3,4,39$ \\
\hline 1743-521 & BPM25114 & 20000 & & & & 1.34 & & & 25 & & & $\mathrm{~m}$ & 5 \\
\hline $1745+607 \mathrm{~J}$ & HS1745+6043 & 35600 & & 8.68 & & 1.05 & & 5400 & & & 120 & & 14 \\
\hline $1748+708$ & GR372 & 6550 & 960 & 8.36 & & 0.98 & 0.17 & 6850 & 150 & & 6.1 & $\mathrm{~m}$ & 5,6 \\
\hline $1814+248$ & G183-035 & 7000 & & & & 0.83 & & & 10 & & & $\mathrm{~m}$ & 5 \\
\hline $1829+547$ & GR374 & 6640 & 360 & 8.50 & & 1.02 & 0.12 & 6150 & 120 & & 15.0 & $\mathrm{~m}$ & 5,6 \\
\hline $1900+705$ & $\mathrm{Grw}+70^{\circ} 8247$ & 13540 & 1470 & 8.58 & & 1.09 & 0.07 & 5760 & 230 & & 13.0 & $\mathrm{~m}$ & $5,6,36$ \\
\hline $2010+310$ & GD 229 & 23000 & & & & 1.28 & & & 500 & & & $\mathrm{~m}$ & 5 \\
\hline $2020-425$ & REJ2024-42 & 29028 & 431 & 8.412 & 0.128 & 0.911 & 0.059 & 6878 & & & & & $1 \mathrm{a}, 1 \mathrm{~b}, 3$ \\
\hline 2039-682 & EG140 & 16065 & & 8.444 & & 0.872 & & 6450 & & & & & 13 \\
\hline 2043-635 & BPM13537 & 25971 & & 8.358 & & 0.855 & & 7054 & & & & & 3 \\
\hline $2055+164$ & EUVE J2055+1627 & 38400 & & 8.37 & & 0.85 & & 6940 & & & 104 & & 4,40 \\
\hline $2107-216$ & GR581 & 5830 & & 8.40 & & 0.85 & & 6700 & & & 23.7 & & 6 \\
\hline $2126+191$ & IK Peg & 34320 & 750 & 8.5 & 0.3 & 1.13 & 0.05 & 5890 & & & 50 & $\mathrm{bc}$ & $27,28,41$ \\
\hline $2157+815$ & HS2157+8153 & 10700 & & 8.71 & & 1.05 & & 5200 & & & 35 & & 14 \\
\hline $2220+133$ & PG2220+134 & 22600 & & 8.81 & & 1.10 & & 4700 & & & 50 & & 14 \\
\hline $2246+223$ & EG155 & 10330 & & 8.57 & & 0.97 & & 5890 & & & 19.0 & & 6 \\
\hline $2251-070$ & GR453 & 4580 & & 8.38 & & 0.82 & & 6740 & & & 8.1 & & 6 \\
\hline $2257-073$ & $\mathrm{BD}-07^{\circ} 5906 \mathrm{~B}$ & 37517 & & 8.25 & & 0.92 & & 8290 & & & 111 & $\mathrm{~b}$ & 28 \\
\hline $2303+465$ & PSR B2303+46 & 45000 & & & & 1.1 & & 3000 & & & 2500 & $\mathrm{~b}$ & 42 \\
\hline $2312-024$ & GR554 & 6840 & & 8.41 & & 0.84 & & 6590 & & & 26.7 & & 6 \\
\hline 2348-444J & ESO292-43 & 5400 & & 8.72 & & 1.04 & & 5130 & & & 26.2 & & 6 \\
\hline 2359-434 & EG165 & 8715 & & 8.581 & & 0.956 & & 5770 & & & & & 13 \\
\hline$\theta$ Hya & HR3665 & 28000 & & 8.5 & & 0.83 & & 5900 & & & 40 & $\mathrm{~b}$ & 27 \\
\hline
\end{tabular}

Remarks - (m) magnetic white dwarfs; (b) white dwarfs with companion(s); (c) close binary or multiple systems; (t) triple systems; (p) common proper-motions binaries.

References - (1a) Marsh et al. (1997a); (1b) Marsh et al. (1997b); (2) Vennes et al. (1997); (3) Finley et al. (1997); (4) Vennes (1999); (5) Fabrika \& Valyavin (1998); (6) Bergeron et al. (2001); (7) Bergeron et al. (1992); (8) Reid (1996); (9) Claver et al. (2001); (10) Bergeron et al. (1995b); (11) Bergeron et al. (1995a); (12) Napiwotzki et al. (1999); (13) Bragaglia et al. (1995); (14) Homeier et al. (1998); (15) Provencal et al. (1998); (16) Heber et al. (1997); (17) Wickramasinghe \& Ferrario (2000); (18) Schmidt et al. (1992); (19) Wegner et al. (1989); (20) Putney (1997); (21) Bergeron et al. (1994); (22) Barstow et al. (1995); (23) Burleigh et al. (1999); (24) Ferrario et al. (1997); (25) Jordan \& Burleigh (1999); (26) Holberg et al. (1998); (27) Burleigh (1999); (28) Vennes et al. (1998); (29) Barstow et al. (1997); (30) Werner \& Rauch (1997); (31) O'Brien et al. (2001); (32) Wegner et al. (1991); (33) Burleigh et al. (1998); (34) Ferrario et al. (1998); (35) Liebert et al. (1993); (36) Suh \& Mathews (2000); (37) Saffer et al. (1989); (38) Werner (1991); (39) Dupuis \& Vennes (1997); (40) Vennes et al. (1997); (41) Wonnacott et al. (1993); (42) van Kerkwijk \& Kulkarni (1999); (43) Moran et al. (1998). 
(15 objects). We believe, that this single bin demonstrates the existence of a secondary maximum in the mass distribution of massive isolated white dwarfs.

When constructing the histogram in Fig. 1 we arbitrarily assumed that stars with masses located exactly at the edges between bins are counted in the bin with stars of higher masses. I.e. if a star has its mass equal to $1.0 M_{\odot}$, then it is counted to the $1.0-1.05 M_{\odot}$ bin.

We have therefore constructed and overlapped in Fig. 1 a second histogram with a finer $\left(0.01 M_{\odot}\right)$ spacing, which demonstrates the structure of the mass distribution function in the vicinity of the secondary maximum, in the range of $0.96-1.12 M_{\odot}$. The maximum is most likely located at about $1.04 M_{\odot}$.

Of course, the mass distribution determined in this sample has been unintentionally blurred by the fact that the mass determinations were done with different methods. In spite of this, the secondary maximum at $\approx 1.04 M_{\odot}$ is clearly visible in Fig. 1 .

The above results can be immediately be compared to the previous mass distribution by Vennes (1999). Our conclusion that there is secondary maximum in the mass distribution at $1.04 M_{\odot}$ remains in full agreement with the above mentioned paper (see his Fig. 8). However, our results deny the possible existence of a secondary maximum at $1.25 M_{\odot}$. The other qualitative difference between Vennes (1999) and our paper is that we demonstrate the existence of a distinct slope in the mass distribution, when we decrease mass towards $0.8 M_{\odot}$.

We note that the present paper is based on a more numerous collection of massive white dwarfs. We tentatively estimated on the basis of Fig. 8 and Table 7 of Vennes (1999) that the number of white dwarfs with masses $M \geq 0.8 M_{\odot}$ in his paper is of the order 30 , whereas our results are based on a much bigger sample of 112 stars.

\section{Massive magnetic vs. nonmagnetic stars}

\subsection{Distribution of masses}

It is interesting to investigate the differences between the mass distribution of the magnetic and nonmagnetic white dwarfs in our sample. It is well established that magnetic white dwarfs apparently have a much higher than average mean mass (Liebert et al. 2003; Kawka et al. 2003). The former paper states that the mean mass of magnetic white dwarfs in their sample equals to $0.93 M_{\odot}$, as compared with the average white dwarf mass of $0.60 M_{\odot}$ (Weidemann 1990). We show our mass distributions in Figs. 2 and 3.

The mass distribution of massive magnetic white dwarfs seems to be flat and does not exhibit any particular features, except the absence of stars with masses in the range 1.15-1.2 $M_{\odot}$ (Fig. 2). However, due to the very small numbers of stars in each bin, typically 1-4 stars, this gap most likely is statistically insignificant. One can note, that the only stars with masses exceeding $1.30 M_{\odot}$ are known magnetic white dwarfs (4 stars), and they are shown in Fig. 2.

In contrast, the mass distribution of nonmagnetic white dwarfs clearly shows the secondary maximum of the mass distribution in the single bin of 1.0-1.05 $M_{\odot}$ (Fig. 3). Moreover,

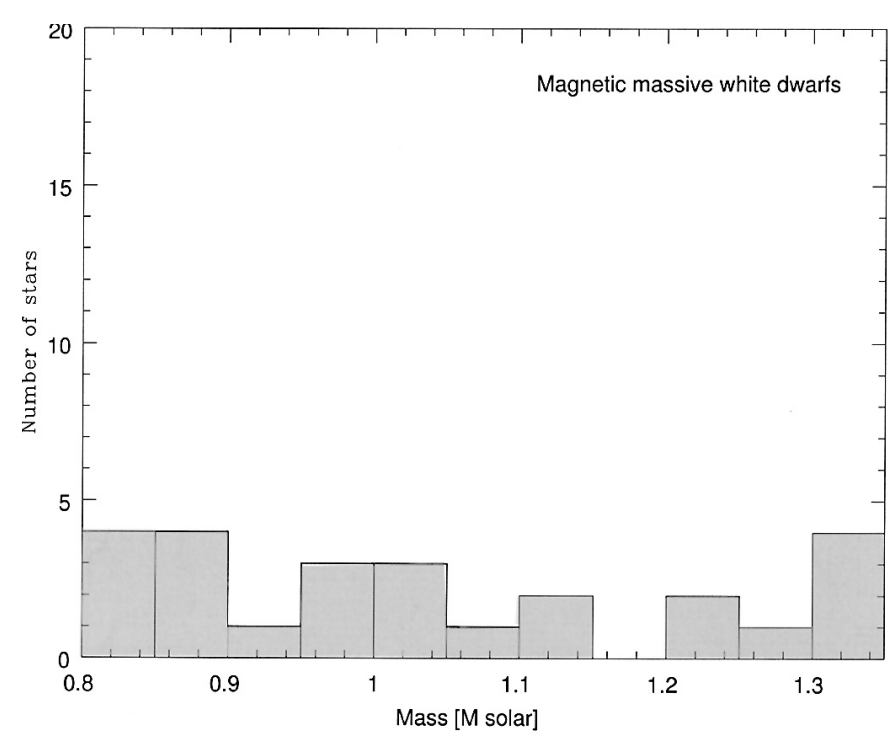

Fig. 2. Mass distribution for magnetic massive white dwarf stars. The mass distribution is flat, and no local maximum can be seen in the figure.

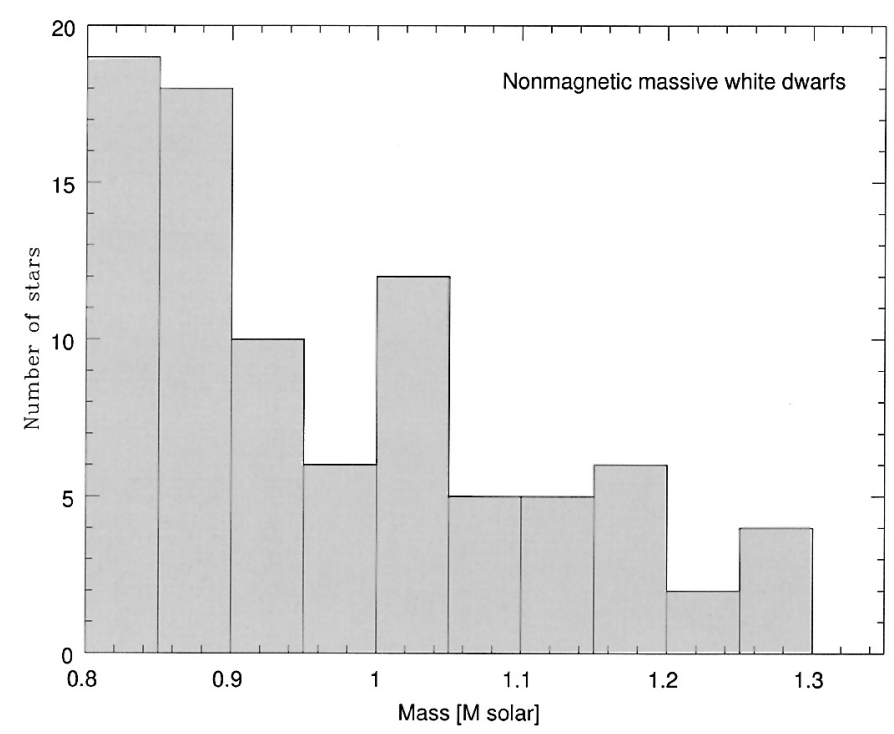

Fig. 3. Mass distribution for nonmagnetic massive white dwarf stars. The local maximum at $1.04 M_{\odot}$, as shown in Fig. 1, should be attributed solely to the above nonmagnetic white dwarfs.

the mass distribution of these stars is qualitatively different from magnetic WDs in that it shows a steeper decrease towards highest masses. In particular, we found no nonmagnetic white dwarf with mass greater than $1.30 M_{\odot}$.

One cannot rule out the possibility that in future some nonmagnetic white dwarfs in Fig. 3 would move to the histogram in Fig. 2, when a non-zero magnetic field is detected.

We point out here that our mass distribution of magnetic massive white dwarfs (Fig. 2) differs significantly from the distribution by Valyavin \& Fabrika (1999). Both authors claim that the mass distribution of magnetic white dwarf stars exhibits a main maximum at $0.8 M_{\odot}$, and a secondary maximum at $\approx 1.15 M_{\odot}$ (see Fig. 2 in their paper). The two maxima in their 
paper are separated by a deep minimum in the mass distribution at $1.05 M_{\odot}$. Our Fig. 2 does not show any such features.

\subsection{Incidence of magnetism in massive stars}

Table 1 immediately provides an estimate of the relative fraction of magnetic white dwarfs in the whole group of massive white dwarfs with masses greater than $0.8 M_{\odot}$. Among 112 massive stars listed in Table 1 we collected 25 stars that are presently known as magnetic objects. Therefore the fraction of isolated magnetic massive white dwarfs in our sample is $22 \%$.

This result is very similar to the conclusion by Vennes (1999), who found that the fraction of magnetic white dwarfs is approximately equal to $25 \%$ for hot stars with masses exceeding $1 M_{\odot}$. However, we stress the essential difference between both analyzes: the sample of hot massive white dwarfs compiled by Vennes (1999) was derived from the EUVE catalogue of hot stars, whereas our sample is not restricted to hot objects.

The data collected in Table 1 allow us to study the distribution of magnetic white dwarfs in a more detailed way. Figure 4 presents the relative fractions of magnetic white dwarfs as a function of mass. Figure 4 clearly suggests that the incidence of magnetism increases with mass, and reaches $100 \%$ in the highest mass bin, 1.30-1.35 $M_{\odot}$. We are aware, however, that the numbers of magnetic stars considered are low, and we cannot exclude strictly random fluctuations.

\subsection{The evolution of magnetic fields in white dwarfs}

We have also attempted to examine the number distribution of massive white dwarfs with respect to effective temperature. Obviously such a distribution has a direct connection to the cooling age of white dwarfs. Figure 5 displays the number distribution of both magnetic (dark tone) and nonmagnetic white dwarfs (light tone) from our Table 1 of massive objects. We remind the reader that we selected 25 magnetic and 87 nonmagnetic massive stars to construct the two histograms in Fig. 5.

It is evident that the distribution in both subclasses of white dwarfs is very similar in that both exhibit a sharp maximum for $T_{\text {eff }}$ between 5000 and $10000 \mathrm{~K}$. This result has been presented also in Valyavin \& Fabrika $(1998,1999)$. However, they suggested that the frequency of cool magnetic stars (of any mass) is greater than that of hot magnetic stars. Our Fig. 5 shows that the distribution of massive magnetic white dwarfs vs. $T_{\text {eff }}$ essentially does not differ from the distribution of all massive objects, therefore effective temperatures below (and also above) $10000 \mathrm{~K}$ do not influence the relative occurrence of magnetism in white dwarfs. In other words, our results show that the occurrence of magnetism in massive white dwarfs does not depend on the cooling age (above $T_{\text {eff }}=5000 \mathrm{~K}$ ).

Figure 5 most probably demonstrates the selection effect that is the reason why we have not yet discovered the majority of the coolest white dwarfs below $T_{\text {eff }}<5000 \mathrm{~K}$, both magnetic and nonmagnetic. We also conclude that our results in Fig. 5 contradict the suggestion of Valyavin \& Fabrika (1998, 1999)

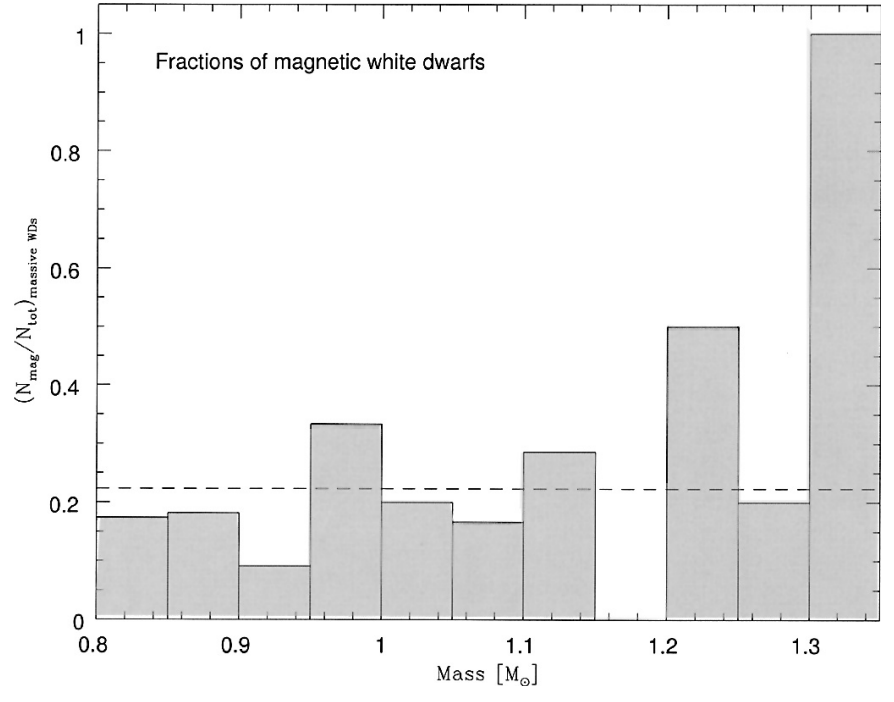

Fig. 4. Relative fractions of magnetic white dwarfs as a function of stellar mass, $N_{\mathrm{mag}} / N_{\mathrm{tot}}$. Note that the incidence of magnetism increases with white dwarf mass. The dashed line denotes the average fraction of magnetic white dwarfs among isolated massive WDs.

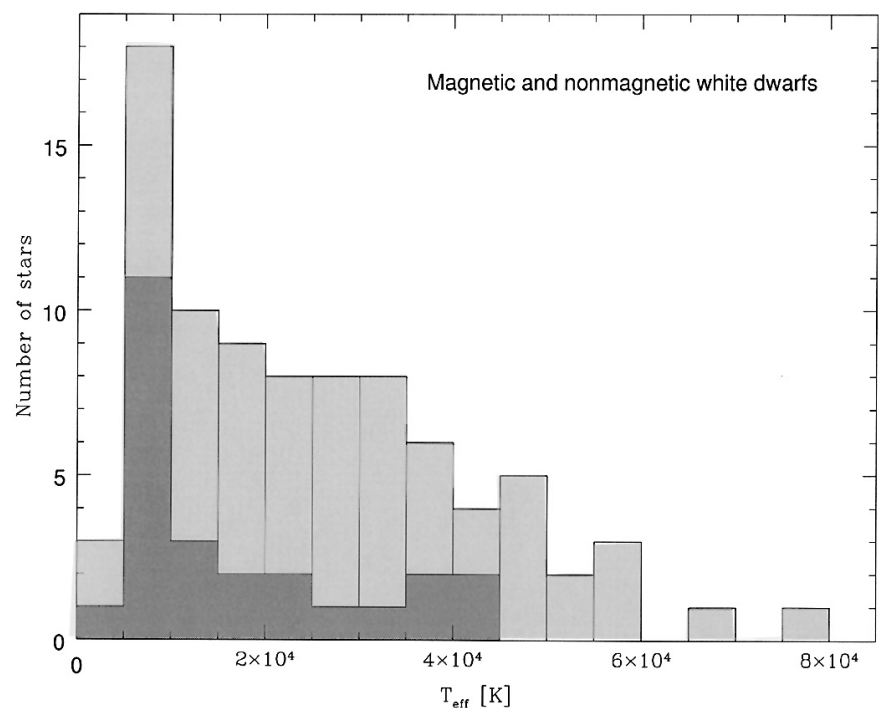

Fig. 5. Number distribution of magnetic (dark tone) and nonmagnetic (light tone) white dwarfs. The distributions of both types of massive white dwarf stars a very sharp maximum in the range $5000 \leq T_{\text {eff }}<10000 \mathrm{~K}$. Computations show that the relative fraction of magnetic white dwarfs vs. $T_{\text {eff }}$ remains almost constant, $0.2<N_{\text {mag }} / N_{\text {tot }}<0.4$ up to $T_{\text {eff }}=40000 \mathrm{~K}$.

that we observe a magnetic field decay below $T_{\text {eff }}<6000$, at least in the group of massive white dwarfs.

\section{Conclusions}

We have performed an extensive search of the available literature and selected all known white dwarf stars of masses at or above $0.80 M_{\odot}$. Included here are stars which are believed to be isolated, or are members of detached (noninteracting) binary systems. We excluded white dwarfs that are members of close (interacting) binary systems. In total, 112 massive white dwarfs 
were selected, and some of them are known as strongly magnetic stars with a surface field $B_{\mathrm{s}}$ approaching 500 megagauss.

The catalogue of all known stellar parameters is presented in Table 1, which lists for each star its parameters $\left(T_{\text {eff }}, \log g\right.$, mass $M$ ) averaged over all the available individual determinations.

The mass distribution of massive magnetic white dwarfs in rather flat, whereas the distribution of nonmagnetic stars seems to be steeper, decreasing towards the maximum Chandrasekhar mass. However, the mass distribution of all massive isolated white dwarfs apparently exhibits a distinct local maximum at $1.04 M_{\odot}$, which seems to be caused exclusively by nonmagnetic white dwarfs in our sample.

We report here the observation that the small group of the most massive stars in our sample, $M>1.30 M_{\odot}$, consists of 4 magnetic white dwarfs. Nonmagnetic white dwarfs apparently are less massive than $1.30 M_{\odot}$.

Our results show that the occurrence of magnetism in massive white dwarfs does not depend on the cooling age (above $\left.T_{\text {eff }}=5000 \mathrm{~K}\right)$.

Acknowledgements. This research has been supported by grant No. 1 P03D 00126 from the Polish Committee for Scientific Research.

\section{References}

Barstow, M. A., Jordan, S., O’Donoghue, D., et al. 1995, MNRAS, 277, 971

Barstow, M. A., Holberg, J. B., Cruise, A. M., \& Penny, A. J. 1997, MNRAS, 290, 505

Bergeron, P., Kidder, K. M., Holberg, J. B., et al. 1991, ApJ, 372, 267

Bergeron, P., Saffer, R., \& Liebert, J. 1992, ApJ, 394, 228 (BSL)

Bergeron, P., Wesemael, P., Beauchamp, A., et al. 1994, ApJ, 432, 305

Bergeron, P., Liebert, J., \& Fulbright, M. S. 1995a, ApJ, 444, 810

Bergeron, P., Wesemael, R., Lamontaigne, R., et al. 1995b, ApJ, 449, 258

Bergeron, P., Leggett, S. K., \& Ruiz, M. T. 2001, ApJS, 133, 413

Bragaglia, A., Renzini, A., \& Bergeron, P. 1995, ApJ, 443, 735

Burleigh, M. 1999, 11th European Workshop on White Dwarfs, ed. S.-E. Solheim, \& E. G. Meistas, ASP Conf. Ser., 169, 249

Burleigh, M. R., \& Barstow, M. A. 1998, MNRAS, 295, L15

Burleigh, M. R., Jordan, S., \& Schweizer, W. 1999, ApJ, 510, L37

Claver, C. F., Liebert, J., Bergeron, P., \& Koester, D. 2001, ApJ, 563, 987

Dupuis, J., \& Vennes, S. 1997, ApJ, 475, L131

Fabrika, S. N., \& Valyavin, G. G. 1998, Bull. Spec. Astrophys. Obs., 45,84

Ferrario, L., Vennes, S., \& Wickramasinghe, D. T. 1998, MNRAS, 299, L1

Ferrario, L., Vennes, S., Wickramasinghe, D. T., Bailey, J., \& Christian, D. J. 1997, MNRAS, 292, 205

Finley, D. S., Koester, D., \& Basri, G. 1997, ApJ, 488, 375

Green, R. F., Schmidt, M., \& Liebert, J. 1986, ApJS, 61, 305

Heber, U., Napiwotzki, R., \& Reid, I. N. 1997, A\&A, 323, 819

Holberg, J. B., Barstow, M. A., Bruhweiler, F. C., Cruise, A. M., \& Penny, A. J. 1998, ApJ, 497, 935

Homeier, D., Koester, D., Hagen, H.-J., et al. 1998, A\&A, 338, 563

van Kerkwijk, M. H., \& Kulkarni, S. R. 1999, ApJ, 516, L25

Jordan, S., \& Burleigh, M. 1999, 11th European Workshop on White Dwarfs, ed. S.-E. Solheim, \& E. G. Meistas, ASP Conf. Ser., 169, 235
Kawka, A., Vennes, S., Wickramasinghe, D. T., \& Schmidt, G. D. 2003, 13th European Workshop on White Dwarfs, ed. R. Silvotti, $\&$ D. de Martino (Dordrecht: Kluwer), in press

Koester, D., Schulz, H., \& Weidemann, V. 1979, A\&A, 76, 262

Koester, D. 2002, A\&AR, 11, 33

Liebert, J., \& Bergeron, P. 1995, in White Dwarfs, ed. D. Koester, \& K. Werner, Lecture Notes in Physics (Springer), 12

Liebert, J., Bergeron, P., Schmidt, G. D., \& Saffer, R. A. 1993, ApJ, 418, 426

Liebert, J., Bergeron, P., \& Holberg, J. B. 2003, AJ, 125, 348

Madej, J., Pietrachowicz, M., Joss, P. C., et al. 2003, A\&A, 412, 837

Marsh, M. C., Barstow, M. A., Buckley, D. A., et al. 1997a, MNRAS, 286,369

Marsh, M. C., Barstow, M. A., Buckley, D. A., et al. 1997b, MNRAS, 287,705

McCook, G. P., \& Sion, E. M. 1999, ApJS, 121, 1

McMahan, R. K. 1989, ApJ, 336, 409

Moran, C., Marsh, T. R., \& Dhillon, V. S. 1998, MNRAS, 299, 218

Napiwotzki, R., Green, P. J., \& Saffer, R. A. 1999, ApJ, 517, 399

O’Brien, M. S., Bond, H. E., \& Sion, E. M. 2001, ApJ, 563, 971

Panei, J. A., Althaus, L. G., \& Benevenuto, O. G. 2000, A\&A, 353, 970

Provencal, J. L., \& Shipman, H. L. 1999, 11th European Workshop on White Dwarfs, ed. J.-E. Solheim, \& E. G. Meistas, ASP Conf. Ser., 169, 293

Provencal, J. L., Shipman, H. L., Hog, E., \& Thejll, P. 1998, ApJ, 494, 759

Putney, A. 1997, ApJS, 112, 527

Reid, N. I. 1996, AJ, 111, 2000

Saffer, R. A., Liebert, J., Wagner, R. M., Sion, E. M., \& Starrfield, S. G. 1989, AJ, 98, 668

Schmidt, H. 1997, in White Dwarfs, ed. J. Isern, M. Hernanz, \& E. García-Berro, Proc. of the 10th European Workshop on White Dwarfs, 3

Schmidt, G. D., Bergeron, P., Liebert, J., \& Saffer, L. 1992, ApJ, 394, 603

Serenelli, A. M., Althaus, L. G., Rohrmann, R. D., \& Benvenuto, O. G. 2001, MNRAS, 325, 607

Serenelli, A. M., Althaus, L. G., Rohrmann, R. D., \& Benvenuto, O. G. 2002, MNRAS, 337, 1091

Shipman, H. L. 1979, ApJ, 228, 240

Suh In-Saeng, \& Mathews, G. J. 2000, ApJ, 530, 949

Rohrmann, R. D., Serenelli, A. M., Althaus, L. G., \& Benvenuto, O. G. 2002, MNRAS, 335, 499

Valyavin, G. G., \& Fabrika, S. N. 1998, Bull. Spec. Astrophys. Obs., 45,69

Valyavin, G. G., \& Fabrika, S. N. 1999, 11th European Workshop on White Dwarfs, ed. S.-E. Solheim, \& E. G. Meistas, ASP Conf. Ser., 169, 206

Vennes, S. 1999, ApJ, 525, 995

Vennes, S., Korpela, E., \& Bowyer, S. 1997a, AJ, 114, 1567

Vennes, S., Thejll, P. A., Galvan, R. G., \& Dupuis, J. 1997b, ApJ, 480, 714

Vennes, S., Christian, D. J., \& Thorstensen, J. R. 1998, ApJ, 502, 763

Wegner, G., Reid, I. N., \& McMahan, R. K. 1989, in White Dwarfs, ed. G. Wegner (New York: Springer), IAU Coll., 114, 378

Wegner, G., Reid, I. N., \& McMahan, R. K. 1991, ApJ, 376, 186

Weidemann, V. 1990, ARA\&A, 28, 103

Weidemann, V., \& Koester, D. 1984, A\&A, 132, 195

Werner, K. 1991, A\&A, 251, 147

Werner, K., \& Rauch, T. 1997, A\&A, 324, L25

Wickramasinghe, D. T., \& Ferrario, L. 2000, PASP, 112, 873

Wonnacott, D., Kellett, B. J., \& Stickland, D. J. 1993, MNRAS, 262, 277 